\title{
MESIODENS EN POSICIÓN INUSUAL: REPORTE DE UN CASO
}

\section{MESIODENS IN UNUSUAL POSITION: A CASE REPORT}

\author{
TITULO CORTO: MESIODENS EN POSICIÓN INUSUAL
}

Martha Rebolledo-Cobos $^{1}$ y Manuel Escalante-Fontalvo ${ }^{1}$

Recibido en Noviembre 26 de 2014

Aceptado en Febrero 05 de 2015

\section{RESUMEN}

Los dientes supernumerarios son alteraciones medianamente frecuentes que aparecen en cualquier área de los arcos dentales y pueden afectar a cualquier órgano dentario anterior, también llamado mesiodens. Esta alteración clínicamente presenta erupción retardada e inclusión de dientes permanentes, apiñamiento, diastemas, mal oclusión y en casos más complejos se pueden encontrar a nivel de piso de fosas nasales o incluídos en el seno maxilar, la mayoría de veces son asintomáticos. Las radiografías panorámica oclusal, peri apical o tomografía computarizada (TC) como ayudas complementarias son de gran utilidad para un buen diagnóstico y ubicación precisa. El tratamiento depende del caso, extracción en casos necesarios o realizar un seguimiento radiográfico. Se presenta un caso clínico del servicio de cirugía oral en la clínica odontológica de la Fundación Hospital Universitario Metropolitano, un paciente de 26 años de edad, masculino con impresión diagnostica de diente supernumerario mesiodens con intento previo de extracción sin éxito. Clínicamente no se evidencian signos de la presencia del mesiodens. Radiografías como ortopantografia, oclusal periapical no permite una posición exacta, por esto se recurre a una tomografía segmentaria logrando mejor ubicación del DS, permitiendo un plan de tratamiento ideal.

Palabras Clave: diente supernumerario; diagnóstico; procedimiento quirúrgico operativo (Decs)

\section{Abstract}

Supernumerary teeth are fairly common disorders that occur in any area of the dental arches and can affect any previous dental organ, also called mesiodens. This disorder presents clinically and inclusion delayed eruption of permanent teeth, crowding, diastema, malocclusion and in more complex cases can be found at ground level nostrils or included in the maxillary sinus, in most cases are asymptomatic. The occlusal panoramic x peri apical or computed tomography (CT) as complementary aids are very useful for proper diagnosis and accurate location. Treatment depends on the case, removal when necessary or perform radiographic follow-up. A case of oral surgery service in the dental clinic of the University Hospital Foundation Metropolitan, a 26 year old male diagnosed with

1. Odontólogos. Especialistas en Estomatología y Cirugía Oral, Universidad de Cartagena. Docentes asistentes Universidad Metropolitana de Barranquilla, Colombia. Correo: malereco18@gmail.com; malereco18@hotmail.com 
print mesiodens supernumerary tooth extraction prior unsuccessful attempt is presented. Clinically, no signs of the presence of mesiodens are evident. Radiographs as Orthopantography occlusal periapical not allow an exact position, so it uses a segmental tomography DS achieving better location, allowing ideal treatment plan.

Keywords: tooth supernumerary; diagnosis; surgical procedures; operative. (Decs-MeSh)

\section{INTRODUCCIÓN}

Los dientes supernumerarios (DS) son alteraciones poco frecuentes que aparecen en cualquier área de los maxilares y, por lo general, su causa de aparición es la duplicación de la lámina dental en la etapa inicial que da la formación de uno o varios órganos dentarios en una o más localizaciones anatómicas. Los DS más comunes son el mesiodens ubicados a nivel de la línea media del maxilar superior, seguido por los premolares ${ }^{1,2}$.

El DS mesiodens representa el 50-80\% del total de ellos, puede presentarse como único o múltiples en un mismo individuo así como coincidir con otros DS o con agenesia de otros dientes. La frecuencia varía entre 0.15$2.65 \%$ y predomina en el sexo masculino, el $80-90 \%$ aparecen en el maxilar superior. La situación más habitual es que se encuentre incluido completamente y solo esté erupcionado en un $25 \%$ de los $\operatorname{casos}^{3-6}$. Se les puede evidenciar en diferentes posiciones como en diversos sitios de la cavidad oral. En casos más complejos se pueden encontrar mesiodens a nivel de piso de fosas nasales 0 incluídos en el seno maxilar ${ }^{7-12}$.

Celikoglu, M. et al., ${ }^{13}$ realizaron una revisión en pacientes con hiperodoncia en los que utilizaron como ayudas diagnósticas imagenológicas: radiografías periapicales y panorámica; reportaron 42 pacientes con casos de hiperodoncia de los cuales el $31.3 \%$ fueros mesiodens seguido por premolares en un $25.0 \%$, laterales $22.9 \%$, disto molar $14.5 \%$ y para molar $4.2 \%, 14$.

Para el diagnóstico es muy importante el aspecto clínico y radiográfico, porque pueden ocasionar erupción retardada e inclusión de dientes permanentes como el incisivo central, apiñamiento y mal oclusiones; la mayoría de los casos son asintomáticos ${ }^{15,12}$, la elección del tipo de radiografía para su correcto diagnóstico pasará por decisión del profesional en el área, entre las ayudas imagenológicas utilizadas se encuentra la radiografía panorámica, periapical, oclusal y la TC, los diagnósticos diferenciales involucran odontomas u otras lesiones radiopacas de los maxilares ${ }^{16,17}$.

En cuanto al tratamiento es muy importante determinar la realización de exodoncia quirúrgica o seguimiento clínico-radiográfico, en la primera opción terapéutica se debe evaluar si el DS impide la erupción del diente permanente o temporal, genera desplazamiento de los dientes adyacentes, sintomatología y si existe patología asociada; en la segunda opción en la cual se decide no realizar la extracción dental y llevar un seguimiento ${ }^{12}$.

Es primordial que el odontólogo conozca los factores etiológicos, características clínicas y las ayudas imagenológicas para el correcto diagnóstico y tratamiento de los dientes supernumerarios. De lo contrario podría cometer errores en el diagnóstico de posición que llevarían a un mal abordaje quirúrgico y complicaciones.

\section{CASO CLÍNICO}

Paciente masculino de 26 años de edad quien acude a la clínica odontológica de la Fundación Hospital Universitario Metropolitano, para la extracción de un DS localizado en el maxilar asintomático. Sin antecedentes familiares relevantes para el caso, manifiesta que fue intervenido quirúrgicamente para la exodoncia del mesiodens cuatro meses previo a la consulta actual, pero no se cumplió el objetivo; reporta además que el abordaje que se le practicó fue por palatino generando dificultad en la apertura bucal, edema e incapacidad. Al examen clínico intra-oral no se evidenció hallazgo y en las radiografías panorámicas, oclusal y periapical se observó zona radiopaca con bordes difusos, localizado en piso de fosas nasales del lado derecho, con un tamaño aproximado de $15 \mathrm{~mm}$ de diámetro, compatible con una estructura dental; (Figura 1). Por la posición inusual y el compromiso de fosas nasales se ordenó TC segmentaria anterior, en la cual se observó imagen radiopaca semejante a un diente invertido, con la corona ubicada hacia piso de fosas nasales y la raíz hacia zona vestibular del maxilar, 
en la reconstrucción tridimensional se observó bien definida la corona del diente comprometiendo el piso de fosas nasales y el desgaste de la misma realizado en la anterior intervención. (Figura 2).

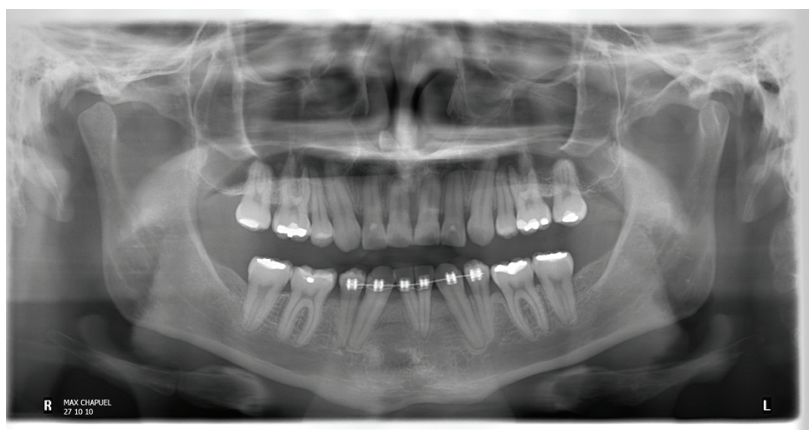

Figura 1. Radiografía panorámica digital que evidencia diente supernumerario en relación a piso de fosas nasal derecha y raíces de órgano dental 11.

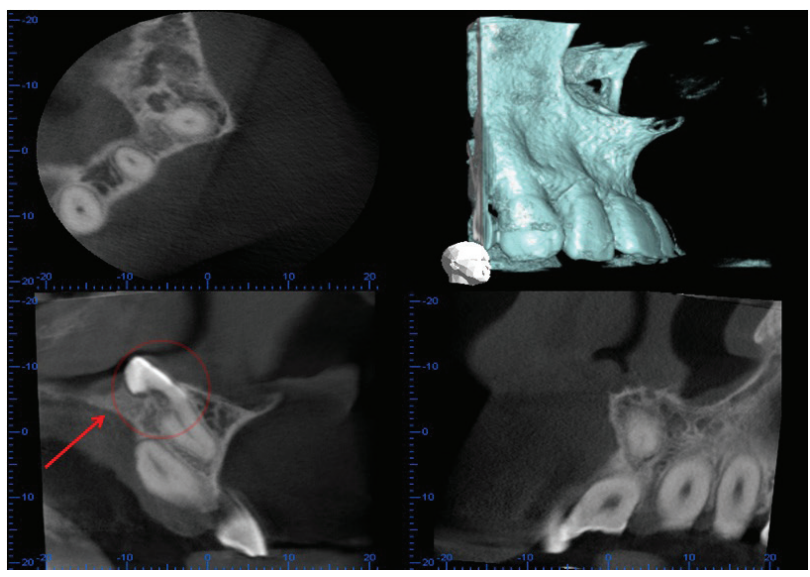

Figura 2. Tomografía segmentaria anterior del maxilar, que evidencia la posición vertical y profundidad del órgano dental supernumerario mesiodens en relación con fosa nasal derecha y raíces de órganos dentales centrales superiores.

Se realizó junta quirúrgica en la cual se decidió realizar un nuevo procedimiento, previo a la firma de un consentimiento informado donde se le explicó al paciente las posibles complicaciones y riesgos, se aplicó técnica anestésica infraorbitaria e infiltración de piso de fosas nasales extra oralmente del lado derecho y a nivel de espina nasal logrando un bloqueo de la zona; se realizó incisión lineal en fondo de surco y se levanta colgajo mucoperiostico, exponiendo el piso de la fosa nasal, posteriormente al liberar la membrana del piso de la fosa nasal, se observó la corona del mesiodens incluido, se realizó osteotomía alrededor de la corona y odontosección, seguidamente con elevador recto apical se efectuó la extracción, primero de la corona dental y después la porción radicular.(Figura 3 y 4). Finalmente se realiza hemostasia e irrigación del área quirúrgica con suero fisiológico, se confrontan los bordes incididos con puntos simples utilizando Vicryl 3-0, se establece terapia antibiótica, con Amoxicilina cápsula de 500mg, una cada 8 horas por 7 días y analgésica antiinflamatoria con Nimesulide tabletas de 100mg una cada 12 horas por 4 días, además terapia con corticoides, Dexametasona ampolla de $8 \mathrm{mg}$ única dosis. En el control postquirúrgico realizado a los 8 días, se observó buen proceso de cicatrización ${ }^{18,19}$.

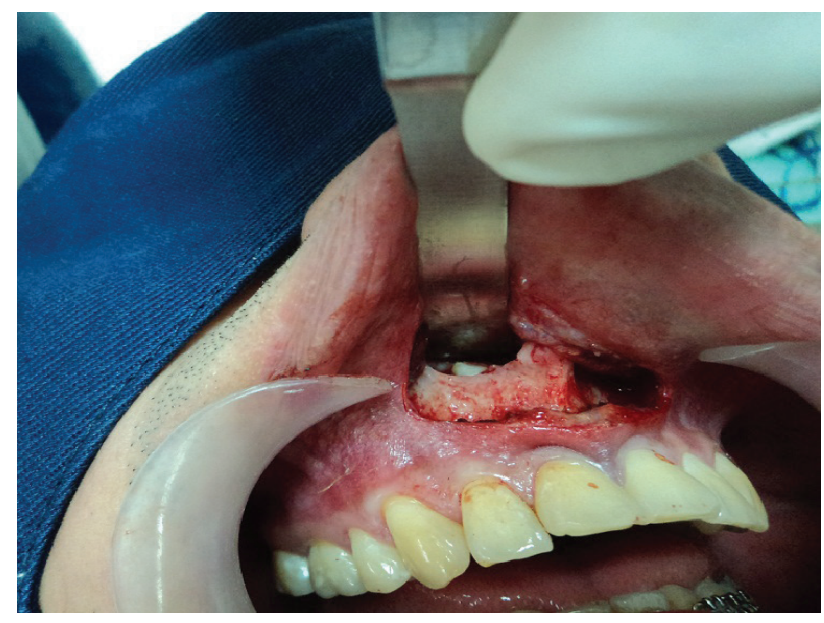

Figura 3. Imagen intraoperatoria que muestra corona de órganos dental supernumerario mesiodens en relación con el piso de la fosa nasal.

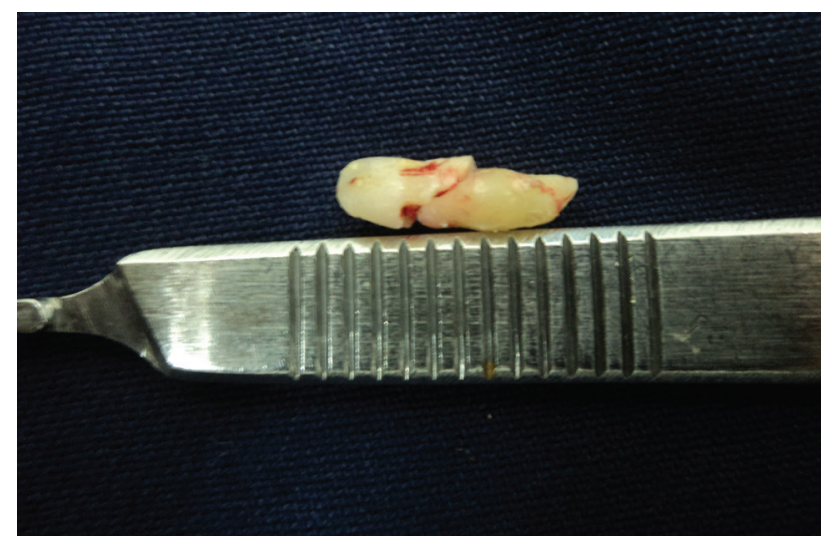

Figura 4. Imagen macroscópica del órgano dental extraído. 
Se obtuvo el consentimiento informado por parte del paciente para la utilización y publicación de la información acompañada de imágenes con fines académicos y científicos.

\section{DISCUSIÓN}

El DS se considera una importante anomalía de la dentición decidua y permanente. Esto es muy preocupante para el odontólogo y familiares del paciente debido que puede causar retardo en la erupción dental y por ende problemas estéticos y funcionales. El diagnóstico de DS es fundamental, así las complicaciones pueden evitarse o minimizarse. Clínicamente la presencia de DS se determina por varios signos clínicos como retardo en la erupción de dientes permanentes, diastemas, apiñamientos. Teniendo en cuenta lo anterior el caso que se reportó no presentó ninguno de los signos clínicos ya que fue encontrado de manera casual ${ }^{20,15,21-23}$.

Los DS tienen predilección por el sexo masculino que el femenino, estudios reportados por Schmuckli et al ${ }^{24}$, reportan que la prevalencia de supernumerarios es mayor en el hombre con porcentaje $1.1 \%$ hombre y $0.4 \%$ mujer. Ferres et $\mathrm{a}^{14}$, realizó estudios en 113 pacientes donde halló 79 dientes supernumerarios en pacientes pediátricos en una edad de 5 a 19 años, manifestó que el $51 \%$ eran hombres y $28 \%$ mujeres donde los supernumerarios mesiodens predominaron en un $56,16 \%$.

Las complicaciones más comunes de la ejecución de los procedimientos quirúrgicos va en relación a la no elaboración de un plan de tratamiento de acuerdo a las imágenes diagnósticas y a la ubicación errónea del DS en el maxilar, debido a esto son recomendables las ayudas como la TC segmentaria, que para el caso mencionado anteriormente, permitió tener claridad y certeza de la ubicación del órgano dental a extraer para diseñar un plan de tratamiento adecuado que incluye un colgajo más conservador, una osteotomía menos agresiva y menos complicaciones ${ }^{16}$. El abordaje seleccionado en el presente caso minimizó el riesgo quirúrgico, mejoro el campo del operador y facilito la extracción de este órgano dental en posición inusual.

\section{REFERENCIAS BIBLIOGRÁFICAS}

1. Mukhopadhyay S. Mesiodens: a clinical and radiographic study in children. J Indian Soc Pedod Prev Dent. 2011;29(1):34-8.
2. Khandelwal V, Nayak AU, Naveen RB, Ninawe N, Nayak PA, Sai Prasad SV. Prevalence of mesiodens among sixto seventeen-year-old school going children of Indore. J Indian Soc Pedod Prev Dent. 2011;29(4):288-93.

3. Díaz ML, Vicente M. Mesiodens: presentación de tres casos. Rev Pediatr Aten Primaria [revista en la Internet]. 2010 Mar [citado 2012 Mayo 09] ; 12(45): 79-87.

4. Kazanci F, Celikoglu M, Miloglu O, Yildirim H, Ceylan I. The frequency and characteristics of mesiodens in a Turkish patient population. Eur J Dent. 2011;5(3):361-5.

5. Russell KA, Folwarczna MA. Mesiodens--diagnosis and management of a common supernumerary tooth. J Can Dent Assoc. 2003;69(6):362-6.

6. Fardi A, Kondylidou-Sidira A, Bachour Z, Parisis N, Tsirlis A. Incidence of impacted and supernumerary teeth-a radiographic study in a North Greek population. Med Oral Patol Oral Cir Bucal. 2011;16(1):e56-61.

7. Asaumi JI, Shibata Y, Yanagi Y, Hisatomi M, Matsuzaki $\mathrm{H}$, Konouchi $\mathrm{H}$, et al. Radiographic examination of mesiodens and their associated complications. Dentomaxillofac Radiol. 2004;33(2):125-7.

8. Montenegro PF, Castellón EV, Aytés LB, Escoda CG. Retrospective study of 145 supernumerary teeth. Med Oral Patol Oral Cir Bucal. 2006 ; 1;11(4):E339-44.

9. Jaspers G, van Gool L. [A man with pain in his upper jaw]. Ned Tijdschr Geneeskd. 2011;155(18):A2391.

10. Van Buggenhout G, Bailleul-Forestier I. Mesiodens. Eur J Med Genet. 2008;51(2):178-181.

11. Gunduz K, Celenk P, Zengin Z, Sumer P. Mesiodens: a radiographic study in children. Journal of oral science. 2008;50(3):287-291.

12. Meighani G, Pakdaman A. Diagnosis and management of supernumerary (mesiodens): a review of the literature. J Dent (Tehran). 2010;7(1):41-9.

13. Celikoglu M, Kamak H, Oktay H. Prevalence and characteristics of supernumerary teeth in a nonsyndrome Turkish population: associated pathologies and proposed treatment. Med Oral Patol Oral Cir Bucal. 2010;15(4):e575-8.

14. Ferres-Padro E, Prats-Armengol J, Ferres-Amat E. A descriptive study of 113 unerupted supernumerary teeth in 79 pediatric patients in Barcelona. Med Oral Patol Oral Cir Bucal. 2009; 1;14 (3):E146-52.

15. Alacam A, Bani M. Mesiodens as a risk factor in treatment of trauma cases. Dent Traumatol. 2009 Apr;25(2):e25-31.

16. Hyun HK, Lee SJ, Lee SH, Hahn SH, Kim JW. Clinical characteristics and complications associated with mesiodentes. J Oral Maxillofac Surg. 2009 ;67(12):2639-43.

17. Gosselin ML, Doyle T, MacLellan J, Anderson RD, Dyment H. A talon cusp mistaken for a mesiodens: case report. J Can Dent Assoc. 2012;78:c6. 
18. Baxendale BR, Vater M, Lavery KM. Dexamethasone reduces pain and swelling following extraction of third molar teeth. Anaesthesia. 1993;48(11):961-4.

19. Graziani F, D’Aiuto F, Arduino PG, Tonelli M, Gabriele M. Perioperative dexamethasone reduces post-surgical sequelae of wisdom tooth removal. A split-mouth randomized double-masked clinical trial. Int J Oral Maxillofac Surg. 2006;35(3):241-6.

20. Chan MY, Shifteh K, Shanske AL. Mesiodens, a new microform of holoprosencephaly? American journal of medical genetics Part A. 2009;149A(2):268-71.

21. Srivatsan P, Aravindha Babu N. Mesiodens with an unusual morphology and multiple impacted supernumerary teeth in a non-syndromic patient. Indian J Dent Res. 2007;18(3):138-140.
22. Ramsaran AS, Barclay S, Scipio E, Ogunsalu C. Nonsyndromal multiple buried supernumerary teeth: report of two cases from the English-speaking Caribbean and a review of the literature. West Indian Med J. 2005;54(5):334-336.

23. Batra P, Duggal R, Parkash H. Non-syndromic multiple supernumerary teeth transmitted as an autosomal dominant trait. J Oral Pathol Med. 2005;34(10):621-625.

24. Schmuckli R, Lipowsky C, Peltomaki T. Prevalence and morphology of supernumerary teeth in the population of a Swiss community. Short communication. Schweiz Monatsschr Zahnmed. 2010;120(11):987-993.

Para citar este artículo: Rebolledo-Cobos M, Escalante-Fontalvo M. Mesiodens en posición inusual: reporte de un caso. Duazary. 2015 jun; 12 (1): 64 - 68 